\title{
Development of reactor relevant pellet launching system technology on ASDEX Upgrade
}

\author{
B. Ploeckl, M. Dibon, P.T. Lang, V. Mertens, W. Treutterer and ASDEX Upgrade Team \\ Max Planck Institute for Plasma Physics, 85748 Garching, Germany
}

\begin{abstract}
Controlling the plasma density in a future fusion reactor will be mainly attributed to pellet injection using a control algorithm based on a rather difficult density measurement. The underlying technology to capacitate the Pellet Launching System (PLS) for the requirements is challenging. The ASDEX Upgrade (AUG) PLS was retrofitted for this task, intensifying the integration into the Discharge Control System (DCS). Lessons learnt and their consequences for the design of a new system "from scratch" will be described.

The technology of the ice production process is discussed as well as the observed performance in view of isotope ratio accuracy and plasma fuelling performance, mimicking the reactor fuel (mixture $\mathrm{D} / \mathrm{T}$ ) by using a mixture of H/D. Since a fusion power plant will require a steady-state pellet source, the development of a control strategy in view of process control is mandatory. The procurement of a new pellet source was launched in order to enhance the existing centrifuge acceleration system. This contribution will show first considerations.

An innovative conceptual design will be presented comprising the potential to replace nowadays centrifuge systems. Present-day vacuum rotating feedthrough technology enables the installation of the motor for the accelerating arm on the atmosphere side avoiding a series of technical difficulties.

The existing PLS on AUG is in operation now for almost 30 years. A conceptual design for a new PLS consisting of extruder, launcher and control systems (also for density control) with focus on reactor relevant technologies except tritium compatibility is under preparation.

Results presented here are complementary domestic activities to the EUROfusion WP TFV.

Keywords: DEMO, Tokamak, pellet fuelling,
\end{abstract}

\section{Introduction}

Future fusion power plants will need suitable fuelling systems, able to operate with boundary conditions given by technology, economic and regulatory issues. Pellet injection into fusion plasmas has been assessed to be the prime choice for fuelling purposes [1] (Lang; SOFT2014). Experiments on nowadays machines are at present mostly focussed on scenario development, technology investigation gets gradually more attention.

\section{Feed-back control of pellet flux rate}

\subsection{Plasma density control}

Future fusion power plants may operate in high confinement mode (H-mode) in order to maximize the harvest of fusion power. The plasma density profiles are strongly peaked and the core density is close or even beyond the Greenwald density $\mathrm{n}_{\mathrm{Gw}}$, the edge density must be kept below a critical level.

Pellet injection disturbs the measurement of the plasma density in high-density plasma discharges due to the fast density increase. On AUG, we use the Bremstrahlung signal as relevant signal for the control loop [2]. The laser interferometer signal (DCN) turned out to be prone to fail due to undetected fringe jumps [3].

\subsection{Connection to DCS}

The pellet centrifuge is a continuous revolving device; hence, the pellet repetition rate is necessarily an integer fraction of the centrifuge frequency. The latter defines the pellet speed, which cannot be changed within a pellet train. Hence, the possible pellet repetition rates are connected to the pellet speed. In the feed forward operation mode, up to five pellet repetition rates are predefined in the controller and available for execution in a pre-determined and time based sequence, see fig. 1. Using the feedback mode, the DCS requests one of these particular predefined repetition rates, the PLC acts accordingly.

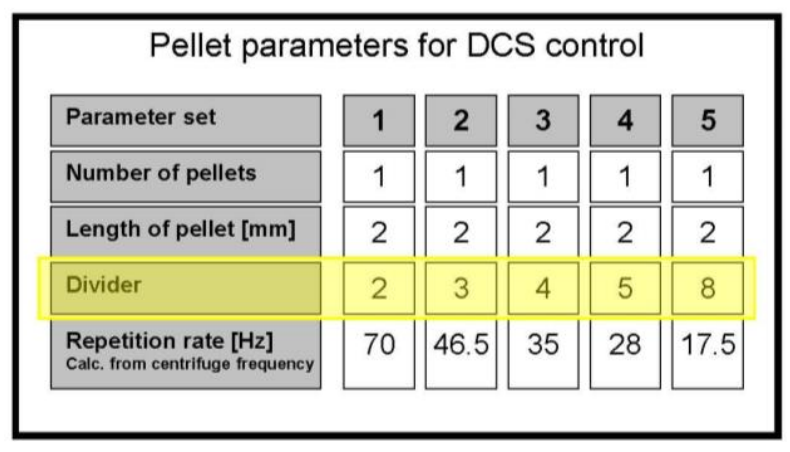

Fig. 1: Example for the predefined data set for DCS control mode at centrifuge frequency of $140 \mathrm{~Hz}$. In feed-back mode, the control parameter is the pellet repletion rate, defined by the divider and selected via the number of parameter set. The number of pellets is set to 1 for each parameter set in this operational mode.

The transition from one parameter set to the next is executed after the full execution of the sequence of the predecessor. One sequence consists of: a) feed of the ice rod (needs $8 \mathrm{~ms}$ for fuelling size pellets with length of $2 \mathrm{~mm})$, b) cut the pellet from the ice rod $(4 \mathrm{~ms})$ synchronized with the centrifuge position and c) the idle time, which defines the cycle time respectively the repetition rate [4].

Setting the idle time to zero defines the maximum possible pellet repetition rate of $83 \mathrm{~Hz}$. Special PLC modules are capable to handle such fast processes having a cycle time in the range of a few microseconds. (Highspeed Boolean Processor FM 352-5).

The actual values of the possible repetition rates are provided to a database and read by the DCS. The PLC of 
PLS receives the request from the DCS and decodes it to one of the predefined data set. Usually dividers of $2 / 3 / 4 / 5 / 8$ were selected corresponding to repetition rates of $70 / 46.5 / 35 / 28 / 17.5 \mathrm{~Hz}$ at a centrifuge frequency of $140 \mathrm{~Hz}$. Zero operation is possible as well.

The PLS provides a return signal to the DCS $3 \mathrm{~ms}$ before the arrival of a pellet on the plasma.

The pellet flight time is derived from the centrifuge speed taking into account constant values (pellet cutting) and speed dependent processes (acceleration). Due to the low time jitter of acceleration process using a centrifuge, the pellets arrive well in the announced time window. The DCS uses this signal e.g. for notching the ECRH in order to avoid beam cut off. The full pellet production cycle must be finished prior the PLS accepts a new parameter set, this makes the return from low repetition rates to faster ones slower than the other way round.

\subsection{Results}

The system is operational and reacts accordingly. The control loop consisting of density measurement, control algorithm and PLS operates reasonable well, however further improvements are necessary and on the way.

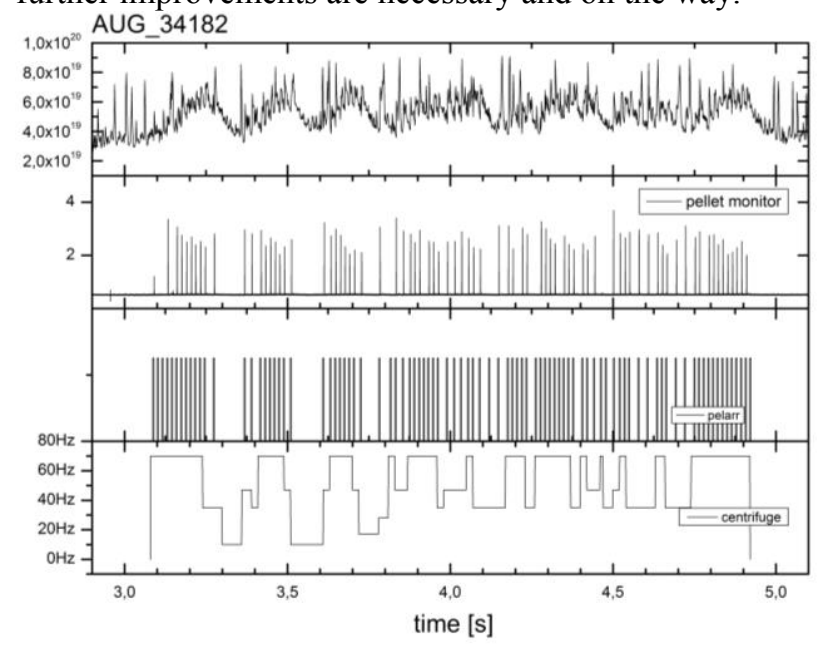

Fig. 2: Feed-back control of core density using Bremstrahlung as controlled process variable (first trace). The actuating variable is the pellet repetition rate requested by the DCS (forth trace). The second trace indicates the pellet ablation signal, which records the emitted light. The third trace displays the record of the pellet arrival signal which announce the arrivals of a pellet on the plasma. The signal is TTL, hence of constant intensity. The pellet survival rate can be evaluated comparing these two traces.

\subsection{Further improvement}

Further enhancement of the system performance will be obtained by creating a new control algorithm for the pellet source. The principle of predefined repetition rates will be abandoned and replaced by a more flexible concept. The rotating centrifuge provides a pellet launch opportunity once a turn. The PLC can claim this timeslot and launch a pellet. During the following $12 \mathrm{~ms}$, no free time-slot will be available, during this period, the requested pellet is produced and the subsequent one is prepared. Hence, the maximum repetition rate is $83 \mathrm{~Hz}$. Doing so, the system can react much faster to changes of the repetition rate in particular from low rates to higher ones. This concept will be extended for the use of the second centrifuge arm and multiple extruders, e.g. one for fuelling and one for ELM pacing pellets. [5] (Lang this conference)

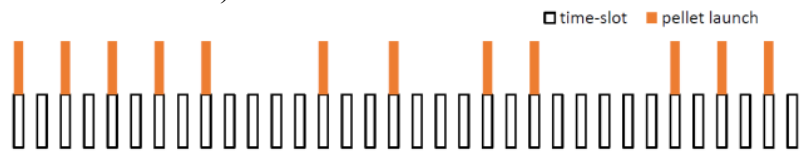

Fig. 3 Schematic view to time-slots from centrifuge running at $140 \mathrm{~Hz}$ (cycle duration $7 \mathrm{~ms}$ ). A minimum distance of $12 \mathrm{~ms}$ leads to the maximum pellet repetition rate of $70 \mathrm{~Hz}$.

\section{Hydrogen isotope mixtures}

\subsection{Motivation}

The burning plasma in future fusion devices will consist of deuterium (D) and tritium (T). Hence, both isotopes have to be continuously fuelled during the discharge. Only cryogenic pellet injection has sufficient efficiency for this process. In view of process control, it seems to be favourable to inject pellets made from a mixture of these both isotopes, most probably in a mixture of $1: 1$. For DEMO, a concept that circulates the fuel from the exhaust of the vacuum vessel pumps directly to the matter injection systems is proposed. This concept was introduced under the name Direct Internal Recycling (DIR) [6]. The required isotope separation and removing of impurities in the redirected gas will be incomplete; otherwise, it will be more difficult to meet regulatory requirements and the costs will exceed the economical limit. Hence, the pellet system must be capable to produce and handle pellets from a mixture of deuterium and tritium with a small amount of protium as well as the corresponding isotopologues (HD, DT, HT).

The presented experiments were performed with protium $(\mathrm{H})$ and deuterium $(\mathrm{T})$ mimicking the $\mathrm{D} / \mathrm{T}$ situation in DEMO [7].

\subsection{Ice production process}

Pellet production systems start with the liquefaction and subsequent freezing of the fuel. This frozen material must be extruded to a rod from which the pellets will be produced. For both processes, the temperature is the main parameter to be controlled. These temperatures must be experimentally investigated in order to design appropriate extrusion systems. On batch extruders like we use at AUG, the freezing of the gas mixture is made at maximum cooling capacity, this leads to a reliable freezing of the material. An isotope anisotropy seems to be likely, e.g. generated by some cryo- distillation processes during gas inlet. Prior to the extrusion the ice made from isotope mixture must be warmed up in order to make the viscosity low enough for the extrusion through the extrusion nozzle. This is a very sensitive process. It turned out, that the optimal extrusion temperature for $\mathrm{H} / \mathrm{D}$-ice is close to that one made of pure deuterium $(\sim 15 \mathrm{~K})$. This is astonishing as the triple point temperature of $\mathrm{H}_{2}$ is $13.9 \mathrm{~K}$ but $18.7 \mathrm{~K}$ for $\mathrm{D} 2$. However, the operation range for this temperature is significantly smaller, indicated by a much stronger temperature dependence of ice viscosity. One possible explanation for that observation is that the protium is frosted within the deuterium bulk. 
The piston batch extruder we used for these tests delivers routinely pellets to AUG, predominantly made from deuterium.

\subsection{Results}

In order to benefit from the advantage to inject both isotopes at once, the isotope distribution in the ice must be known. Ideally, it is according to the isotope ratio in the gas mixture, the ice is produced from and constant over the length of the ice rod. An analysis was performed in order to verify this assumption.

For this, we pumped the torus of AUG to the background pressure; closed the gate valves to the turbopumps and launched pellets in the now unpumped vacuum vessel where they are smashed on its opposite first wall (without plasma). The isotope ratio is determined using residual gas analysis (RGA) with quadrupole mass spectrometer. For each pellet train, consisting of 3 pellets, the isotope ratio is determined. After 30 pellets, the vacuum vessel reached the pressure limit for RGA operation and is pumped down again to recover sufficient vacuum conditions and the procedure restarts from beginning.

Fig. 2 shows the obtained isotope ratio for tree ice rods. The ratio is $1: 1$ within the error margins. The distribution is considered homogeneous.

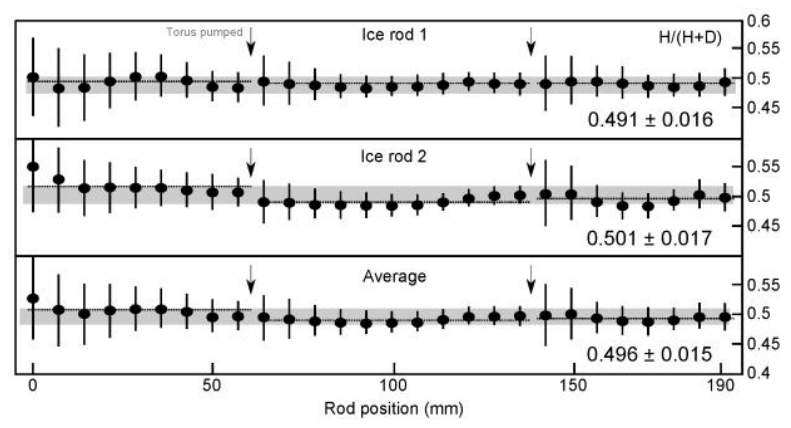

Fig. 4: H/D Isotope ratio along ice rods measured by analysing pellet trains of 3 pellets launched into the not pumped vacuum vessel of AUG. The isotope distribution is close to unity within the error margins.

\subsection{New pellet source}

Recently, we started activities to develop a new pellet source for AUG. The current pellet source is able to deliver 95 fuelling pellets $(1.9 \times 1.9 \times 2 \mathrm{~mm})$ or 125 pacing pellets $(1.4 \times 1.4 \times 1.5 \mathrm{~mm})$. The present investigations on high density scenarios and feed-back control experiments raised the request to provide more pellets per discharge in order to cover the whole discharge with pellets at a sufficient repetition rate. For this purpose, about 400 pellets will be sufficient. The maximum amount of pellets in the present system is defined according to inventory limits in order to avoid explosion risks. To overcome this limitation is an important step towards a continuous extrusion system, capable to serve on superconducting machines with much longer pulses than AUG. The main idea is to separate the vacuum of the pellet source from those of the centrifuge [8]. This helps to apply different explosion prevention concepts. For the pellet source volume, the components must be certified that they are no ignition source. The centrifuge part of the system (pellet acceleration) may follow the present concept to avoid explosive atmosphere by the limitation of the concentration of hydrogen even in case of incidence.

\section{New centrifuge drive concept}

\subsection{Rationale and concept}

In the past decades, several concepts for pellet injection systems were developed and tested. There were different concepts realized to produce pellets and to accelerate them [9]. The simplest solution is a pipe gun, which combines the pellet formation in a pipe and the acceleration with a short propellant gas pulse. Extrusion systems are producing an ice rod and some mechanism cut the pellets from this. In these systems, the acceleration stage was based on pneumatic principle (gas gun or blower gun) or mechanic acceleration (centrifuge). Both systems are in use on several fusion devices and have their strength and weakness. The pneumatic principle is simpler and has achieved adequate reliability, which is why most of existing systems are relying this principle.

Several centrifuge systems have been built in the past decades, all with a drive based on turbomolecular pump motors. Presently, just the AUG centrifuge is still in operation. Recent studies showed, that for a future fusion reactor, mechanic acceleration is preferred, as this principle provides the low speed scatter needed for plasma control purposes $[10,11]$.

In order to make technological progress useful, we have put our efforts to get the drive out of the vacuum. Since a couple of years, ferromagnetic feedthroughs are commercially available in a frequency range worth to be investigated. The drive can be then a commercial motor for high speed application, operated at atmosphere.

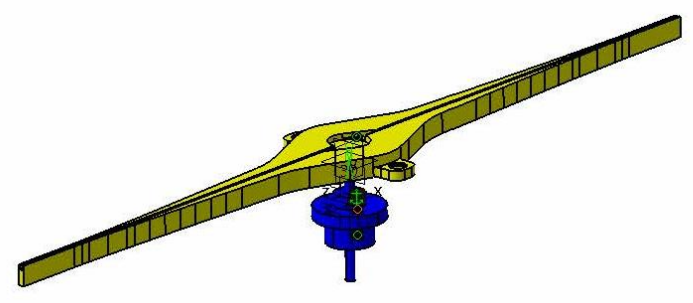

Fig. 5: Centrifuge with rotary feedthrough

Main issue is the suitability for the requested rotation speed and the stability of the bearings for the expected loads. The first attempt is a rotation speed of $10.000 \mathrm{rpm}$ $(167 \mathrm{~Hz})$ according to commercial specification.

\subsection{FEM simulations}

Two main impacts on the bearings are expected: forces due to imbalanced mass and shocks. The relevance of these two events is investigated using FEM method. We use the dimensions of the existing AUG centrifuge arm, supposing that a future optimized arm will be comparable or better.

The ferro-fluidic feedthrough is from ALMA with $10.000 \mathrm{rpm}(167 \mathrm{~Hz})$ maximal rotating speed. The 
maximum axial force is $50 \mathrm{~N}$, the maximum radial force is $75 \mathrm{~N}$.

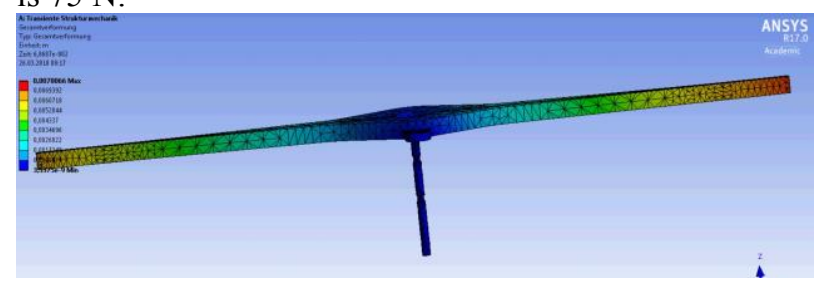

Fig. 6: FEM simlation

The setup is not very sensitive to shocks; the forces to the upper bearing are within the specified range up to a shock with an intensity of $36 \mathrm{kNm}$. Hence, the main issue is to obtain sufficient mass balance.

For the FEM simulations, an additional mass of $1 \mathrm{~g}$ was added on the arm and this mass was varied on its radially position. Hence the value of the imbalance is given in $\mathrm{g} \times \mathrm{mm}$.

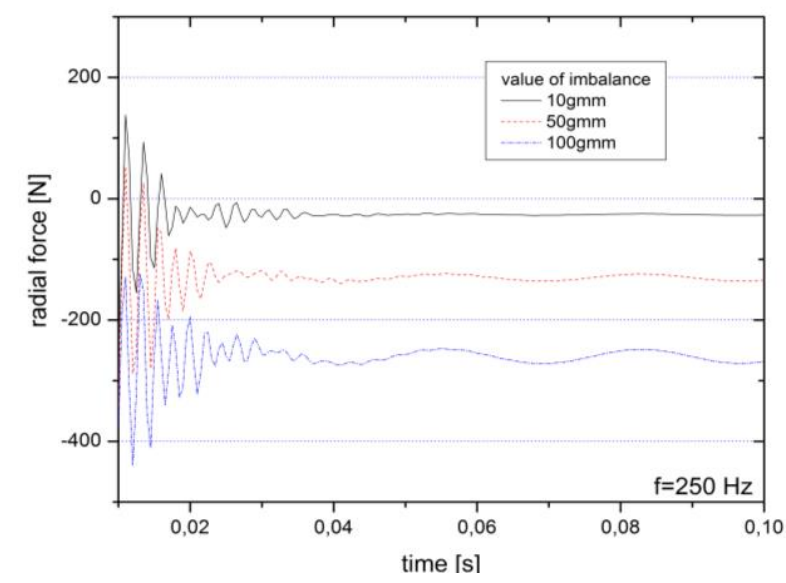

Fig. 7: Trend of the radial forces on the upper bearing of the rotary feedthrough. Oscillations in the first $20 \mathrm{~ms}$ are considered to be numerical artefacts.

The main contribution of the imbalance affects the radial forces on the upper bearing of the feedthrough.

Further activities are planned to develop a sound balancing process.

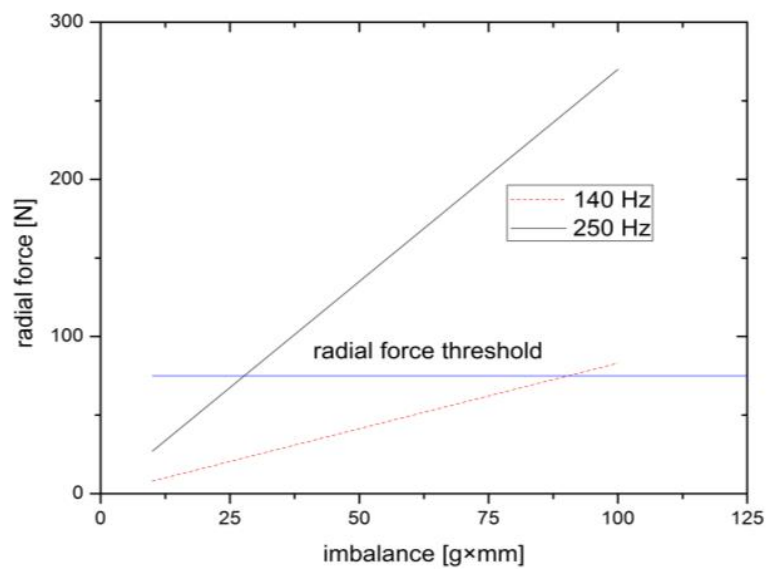

Fig. 8: Radial forces on the upper bearing of the rotary feedthrough evoked by various imbalances. The radial maximum force limit of the upper bearing given by the manufacturer is at $75 \mathrm{~N}$. Hence, the imbalance must be well below $25 \mathrm{~g} \times \mathrm{mm}$ for systems capable for frequencies up to $250 \mathrm{~Hz}$.

\subsection{Next steps}

Next important steps will be to perform dynamic tests in order to evaluate the stability under maximum rotating speed as well as to establish knowledge of balancing techniques.

For future applications, a higher rotation speed is desirable.

\section{Conclusion and outlook}

The availability of reactor relevant pellet technology is an important issue for future fusion devices. In particular for control purposes, the centrifuge acceleration system has strong advantages. It will be very important to develop concepts to combine continuous extruders with centrifuge under nowadays-technical options and safety rules.

The technology to handle isotope mixtures in the fuelling system will be an important issue for DEMO.

\section{Acknowledgement}

This work has been carried out within the framework of the EUROfusion Consortium and has received funding from the Euratom research and training programme 2014-2018 under grant agreement No 633053. The views and opinions expressed herein do not necessarily reflect those of the European Commission.

\section{References}

[1] P.T. Lang, et. Al. Considerations on the DEMO pellet fuelling system (Fusion Engineering and Design 96-97 (2015) 123-128)

[2] T.C. Blanken, et.al., "Control-oriented modeling of the plasma particle density in tokamaks and application to real-time density profile reconstruction" Fusion Engineering and Design 126 (2018), 87 - 103

[3] C.J. Rapson, el al., Experiments on actuator management and integrated control at ASDEX Upgrade, Fusion Engineering and Design 123 (2017) 603-606

[4] B. Plöckl and P. T. Lang, The enhanced ASDEX Upgrade pellet centrifuge launcher; Rev. Sci. Instrum. 84, 103509 (2013)

[5] P.T. Lang, et al., Final design of the JT-60SA pellet launching system for simultaneous density and ELM control, this conference.

[6] Chr. Day, et al., Consequences of the technology survey and gap analysis on the EUDEMO R\&D programme in tritium, matter injection and vacuum, Fusion Engineering and Design 109-111 (2016) 299-308

[7] P.T. Lang, et al. EPS 2018

[8] B. Ploeckl, et al., The enhanced high speed inboard pellet fuelling system at ASDEX Upgrade, Fusion Engineering and Design 88 (2013) 1059- 1063

[9] S. K. Combs \& L. R. Baylor (2018) Pellet-Injector Technology-Brief History and Key Developments in the Last 25 Years, Fusion Science and Technology, 73:4, 493-518

[10] B. Ploeckl, et al., Comparison of different pellet injection systems for ELM pacing, Fusion Engineering and Design 86 (2011) 1022-1025

[11] P.T. Lang, et al, Isotope mixture control in the high density regime by pellet injection at ASDEX Upgrade EPS 2014 Prague 